\title{
HACIA UNA POLÍTICA DE EVALUACIÓN DE FUSIONES HORIZONTALES*
}

\author{
Claudio Agostini
}

\begin{abstract}
Determinar los potenciales efectos de una fusión en el grado de competencia en un mercado es una tarea compleja. La decisión de las instituciones que defienden la libre competencia respecto a aprobar o rechazar una fusión es, por lo tanto, difícil, y genera incertidumbre en las empresas que están evaluando fusionarse. Establecer una política explícita para evaluar fusiones, consistente en un marco analítico para determinar qué tan probable es que una fusión reduzca el grado de competencia en un mercado, cumpliría con dos objetivos: sistematizar el análisis y permitir a los agentes privados anticiparse a las acciones de las instituciones fiscalizadoras, reduciendo así la incertidumbre. Este trabajo propone una política de fusiones para Chile que cumpla con los objetivos anteriores, en línea con políticas aplicadas en países desarrollados.
\end{abstract}

Claudio Agostini. Doctor en Economía, University of Michigan. Profesor asistente, Ilades-Universidad Alberto Hurtado. Email: agostini@uahurtado.cl.

* Agradezco los comentarios de Aldo González, Eduardo Saavedra, Ricardo Sanhueza y un árbitro anónimo de Estudios Públicos. 


\section{Introducción}

Е

n los últimos años hemos observado fusiones importantes de empresas en distintos sectores de la economía. Muchas de ellas han generado una discusión pública importante respecto a sus efectos en la concentración de los mercados y en el grado de competencia en cada uno de ellos. En particular, la discusión se ha centrado en las fusiones que fueron permitidas por los organismos de defensa de la libre competencia (Comisión Resolutiva y posteriormente el Tribunal de la Libre Competencia) y que involucraron bancos, supermercados, empresas telefónicas y empresas de televisión por cable.

La mayor preocupación pública se refiere a si estas fusiones disminuirán significativamente el grado de competencia en los mercados involucrados y, por lo tanto, si éstas deberían haber sido bloqueadas o no (o haber sido aprobadas con condiciones o medidas de mitigación). Responder estas preguntas no es trivial y el debate público ha sido más ideológico que técnico.

En general, no es fácil determinar los potenciales efectos de una fusión en el grado de competencia en un mercado, ya que se debe evaluar la diferencia en el grado de competencia futura en un mercado entre un escenario con fusión y uno sin fusión. Por lo tanto, la decisión de un organismo de defensa de la libre competencia respecto a aprobar o rechazar una fusión es una decisión difícil de tomar y que genera, además, incertidumbre en las empresas que están evaluando la posibilidad de fusionarse.

Una forma de sistematizar el análisis requerido para intentar responder las preguntas anteriores es establecer una política explícita para evaluar fusiones, la cual consiste en un marco analítico que sirva para determinar qué tan probable es que una fusión reduzca el grado de competencia en un mercado.

El objetivo principal de una política de fusiones es diseñar una metodología a ser seguida por las instituciones que deben promover y garantizar la libre competencia. No sólo se trata de sistematizar el análisis para evaluar si una fusión debe objetarse o no, sino también de permitir que los agentes privados puedan anticiparse a las acciones de estas instituciones. En este sentido, una de las ventajas de tener una política explícita es que las empresas que están considerando una fusión enfrentarían un escenario fiscalizador menos incierto.

Una política clara y simple de análisis de fusiones reduciría la incertidumbre y los costos de cumplimiento asociados a la fiscalización e investigaciones realizadas, en el caso de Chile por la Fiscalía Nacional Económica 
(FNE), ya que constituiría el marco analítico que adopta la FNE en su accionar $^{1}$.

Por estas razones, hay varios países desarrollados que han implementado explícitamente una política para evaluar fusiones: Estados Unidos en 1982 (revisada y modificada en 1984, 1992 y 1997); Canadá en 1991; Noruega, Nueva Zelanda y Australia en 1996; Inglaterra en 1999, y la Unión Europea en 2002.

Adicionalmente, en el caso de Chile, la adopción de una política de fusiones obligaría a la FNE a fortalecer los análisis económicos que realiza. La razón es que estaría sujeta a un escrutinio público basado en estándares internacionales originados a partir de la literatura económica, tanto teórica como empírica, de la organización industrial. Éste es un aspecto importante, ya que una buena institucionalidad económica que promueva el crecimiento económico requiere instituciones fuertes que garanticen la competencia en los mercados y si bien en Chile se ha avanzado mucho en el último tiempo, queda camino por recorrer y fortalecer la FNE es un paso importante en ese camino.

En este artículo se propone una política clara y explícita para evaluar fusiones, en línea con los principios económicos utilizados por países desarrollados en sus políticas de fusiones. Con este objetivo, se establece un marco analítico en cuatro etapas.

Primero, se determina el mercado relevante que será afectado por la fusión (puede ser más de un mercado).

Segundo, se evalúa el grado y el aumento en la concentración en el mercado relevante post-fusión para establecer si la fusión puede generar efectos anticompetitivos en el mercado relevante.

Tercero, se evalúa si existe la posibilidad de que haya entrada probable, oportuna y suficiente en el mercado para contrarrestar los potenciales efectos anticompetitivos de la fusión.

Cuarto, se evalúan las ganancias de eficiencia que produce la fusión, si ellas pueden o no conseguirse a través de otros medios distintos a la fusión y si estas ganancias de eficiencia compensan los potenciales efectos anticompetitivos de la fusión (en el caso en que los haya).

Es importante mencionar que una simple aplicación mecánica de la metodología propuesta puede llevar a conclusiones erradas. Se requiere utilizar esta metodología en forma razonable, con flexibilidad y considerando las especificidades de cada fusión e industria en particular.

\footnotetext{
${ }^{1}$ Es importante recalcar que una política para evaluar fusiones no es un marco legal para ser utilizado en los tribunales.
} 


\section{2. ¿Por qué una fusión puede tener efectos anticompetitivos?}

Algunas fusiones pueden generar poder de mercado, distorsionando la asignación de los recursos ${ }^{2}$. En general, hay tres tipos de preocupación con respecto a los potenciales efectos negativos que pueda tener una fusión en el grado de competencia en un mercado: efectos cooperativos, efectos unilaterales y comportamiento estratégico.

\section{a) Poder de mercado cooperativo (colusión)}

Una fusión, al reducir el número de empresas participantes en el mercado, aumenta la probabilidad de que las empresas que quedan en el mercado puedan coludirse y fijar precios superiores a los perfectamente competitivos, ya que coordinarse es más fácil. Esta coordinación puede ser explícita o implícita. En este último caso se requiere el uso de algunos mecanismos facilitadores como señales de precios, paralelismo consciente o liderazgo en precios.

\section{b) Poder de mercado unilateral}

Una fusión que involucra empresas que venden o fabrican productos que son sustitutos cercanos puede relajar la competencia en precios en cualquiera de los dos productos eliminando el sustituto principal, lo cual depende del grado de sustitución entre ambos productos y no de la cooperación entre varios vendedores o productores. La empresa fusionada puede incluso en forma rentable simplemente reducir la producción. Los efectos unilaterales dependen críticamente de la sustitutividad de la demanda entre los productos que producen las empresas que se fusionan (y también respecto a otros productos sustitutos en el mercado).

\section{c) Comportamiento estratégico}

La conducta estratégica ha sido ilustrada en diferentes modelos que muestran empresas actuando racionalmente y que logran subir el costo de sus rivales o cerrarles el mercado. Esto no se debe a un efecto unilateral ni a uno cooperativo, sino a los esfuerzos de una empresa para disminuir los

${ }^{2}$ El premio Nobel George Stigler (1950), por ejemplo, sostiene que el obtener poder de mercado puede haber sido una de las principales motivaciones en las fusiones ocurridas durante los últimos 25 años del siglo XIX y los primeros 50 del siglo XX. Es así como denomina "fusiones para monopolio" a la ola de fusiones ocurrida entre 1887 y 1904 y "fusiones para oligopolio" a la ola de fusiones ocurrida entre 1916 y 1929. 
efectos de la competencia de empresas rivales o para impedir la entrada de un nuevo competidor.

La evaluación de una fusión debería considerar explícitamente si el riesgo principal se debe a efectos unilaterales, efectos cooperativos o a comportamiento estratégico, ya que esto permite determinar el tipo de información requerida y eventualmente poner condiciones al aprobar la fusión.

\section{Mercado relevante}

Para determinar las características de un mercado es necesario, en primer lugar, definir un mercado que económicamente tenga sentido. El objetivo es definir un mercado que pueda ser sujeto de prácticas anticompetitivas o que permita ejercer poder de mercado.

El criterio más utilizado para determinar el mercado relevante, a partir del trabajo conjunto realizado por el Departamento de Justicia y la Comisión Federal de Comercio en Estados Unidos, se basa en definir un mercado en el cual las empresas podrían ejercer poder de mercado si fueran capaces de coordinar sus acciones.

Para estos efectos se utiliza una definición de mercado relevante que se centra únicamente en los factores que influyen en la sustitución de la demanda, es decir, en la respuesta posible de los consumidores frente a un cambio en los precios relativos. La razón para esto es que la posibilidad de que una empresa o un grupo de empresas puedan efectivamente aumentar el precio en un mercado, sin que pierda muchas ventas y dicho aumento termine siendo no rentable, depende fundamentalmente de la disposición que tengan los consumidores para pagar el nuevo precio más alto 3 .

Un mercado se define como un bien (o servicio) o conjunto de productos (o servicios) y un área geográfica en la cual éste es producido o vendido, tal que una empresa hipotética que maximiza sus utilidades, que no está sujeta a regulación de precios y que es la única empresa productora o vendedora de estos productos en el presente y en el futuro en dicha área geográfica, podría imponer un aumento pequeño pero significativo de los precios en forma no transitoria.

Un mercado relevante consiste entonces en un producto o grupo de productos y un área geográfica no mayor que lo necesario para satisfacer la definición anterior.

${ }^{3}$ Las respuestas por el lado de la oferta también son importantes, pero son consideradas posteriormente al definir los participantes en el mercado relevante y las condiciones de entrada. 
Para evaluar si un monopolista hipotético podría estar en condiciones de ejercer poder de mercado es necesario evaluar las reacciones de los consumidores frente a un aumento en los precios. Un aumento no transitorio en los precios puede no ser rentable para el monopolista hipotético si los consumidores comienzan a adquirir productos sustitutos o el mismo producto generado por empresas en otras áreas geográficas.

Si bien este concepto de mercado relevante no tiene validez legal en ningún país de los que explícitamente lo adoptan en su política de evaluación de fusiones, en la práctica, ningún caso en las cortes de Estados Unidos, Canadá, Australia y Nueva Zelanda ha rechazado la implementación de dicha política y ningún caso ha utilizado una definición de mercado relevante que sea contradictoria con el test del monopolista hipotético.

Adicionalmente, es importante señalar que este test del monopolista hipotético no asume ni requiere que la fusión efectivamente tenga el efecto de aumentar los precios, sino que simplemente constituye una herramienta analítica para identificar el mercado relevante.

\section{a) Mercado de producto relevante}

En forma consecuente con la definición anterior, el mercado de producto relevante consiste en un producto o conjunto de productos tal que, una empresa hipotética que maximiza utilidades y que es la única empresa que vende y venderá esos productos, podría sostener en forma no transitoria un aumento de precios pequeño pero significativo (en general, se considera un aumento entre 5 y 10\% durante al menos un año).

En otras palabras, si la única reacción de los consumidores frente a un alza en el precio es cambiarse hacia el consumo de otros productos, para el monopolista hipotético es rentable subir los precios en una magnitud pequeña pero significativa y por un período de tiempo no transitorio.

En la práctica, para definir el mercado de producto relevante se comienza postulando un mercado para cada producto de las empresas que se fusionarían. Para el mercado que se postula como relevante se pregunta si sería rentable para un monopolista hipotético aumentar los precios en forma significativa y no transitoria. Si se concluye que los consumidores se cambiarían a otros productos en número suficiente frente al aumento en el precio como para que dicho aumento no sea rentable para el monopolista, entonces el mercado que se postulaba como relevante no lo es y se debe incluir el producto o servicio sustituto más cercano. El análisis vuelve a repetirse hasta que se encuentra un mercado en el cual el monopolista hipotético puede aumentar rentable y sostenidamente el precio.

En el análisis de mercado de producto relevante uno de los factores más importantes a considerar es la existencia de "costos de cambio" 
(switching costs) para los consumidores, ya que desincentivan que los compradores puedan cambiarse a productos sustitutos cuando enfrentan aumentos en el precio.

\section{b) Mercado geográfico relevante}

De igual forma, un mercado geográfico relevante consiste en un área geográfica tal, que para un monopolista hipotético que es el único vendedor presente y futuro del producto relevante en dicha área geográfica sería rentable aumentar el precio en una magnitud pequeña pero significativa por un período de tiempo no transitorio, mientras los precios de todos los productos vendidos en otras áreas geográficas permanecen constantes.

En otras palabras, si asumimos que la única respuesta de los consumidores al aumento de precios es cambiar su consumo hacia productos que se venden en otras áreas geográficas fuera del área definida, para el monopolista hipotético sería rentable subir los precios en una magnitud pequeña pero significativa y por un período de tiempo no transitorio.

En la práctica el test del monopolista hipotético se aplica igual que en el caso del mercado de producto relevante. Se parte definiendo como mercado geográfico la ubicación más pequeña donde el producto relevante se produce o se vende. Si frente a un aumento en el precio los consumidores comenzaran a comprar el producto de vendedores de otras localidades geográficas en tal magnitud que el aumento en el precio no sea rentable para el monopolista, entonces se debe agregar la ubicación geográfica que es el sustituto más cercano. El proceso continúa hasta encontrar el área geográfica más pequeña donde un monopolista hipotético pueda sostener rentablemente un aumento en los precios.

En el caso del mercado geográfico relevante algunos factores importantes de considerar en el análisis son: los costos de transporte, si el bien o servicio requiere de una interacción cara a cara entre el vendedor y el cliente, el grado de perecibilidad del producto relevante, el ámbito geográfico de la publicidad realizada por los participantes en el mercado, la frecuencia y confiabilidad del servicio de reparto y la existencia de contratos que impidan cambiarse de proveedor.

Finalmente, es importante señalar que si bien al evaluar los efectos de una fusión la principal preocupación se refiere al efecto en los precios y la cantidad en el mercado relevante, hay casos en los cuales otras dimensiones pueden ser más importantes. Existen mercados en los cuales hay niveles significativos de competencia en aspectos distintos del precio, como por 
ejemplo, la calidad, el servicio, la innovación, la publicidad y la variedad de productos. En estos casos es posible implementar el mismo test del monopolista hipotético, pero utilizando, por ejemplo, disminución en la calidad en vez de aumento en los precios.

De igual forma, hay mercados que tienen como característica el que las transacciones son infrecuentes, como en el de bienes durables o mercados con contratos de largo plazo. En estos casos es importante definir bien la dimensión temporal del mercado, ya que frente a un aumento en el precio del bien los consumidores pueden responder, por ejemplo, postergando el reemplazo del bien. Adicionalmente, existen mercados con características temporales (restaurantes a la hora de almuerzo y a la hora de comida) o estacionales importantes (frutas en invierno y en verano) que deben ser consideradas.

Por último, es necesario señalar que el test del monopolista hipotético puede resultar inadecuado si existe una empresa dominante en el mercado. La razón es que la empresa puede haber ya ejercido poder de mercado y aumentado el precio hasta un punto en que ya no resulta rentable subirlo. En este caso, la aplicación del test del monopolista hipotético llevaría a la determinación de un mercado relevante muy amplio ${ }^{4}$. Para solucionar dicho problema se debe aplicar el test considerando el precio competitivo en el mercado en vez del precio existente.

\section{4. ¿Cómo implementar la definición de mercado relevante?}

La idea de considerar un monopolista potencial es relativamente simple, pero útil y poderosa como herramienta analítica, por lo que se ha constituido en una de las piedras fundamentales sobre las cuales se basan las distintas políticas para evaluar fusiones en distintos países.

Si bien la idea no es nueva y había sido planteada explícitamente por Adelman en 1959, Posner en 1976 y Sullivan en 1977, es a partir de los “Merger Guidelines” de 1982, del Departamento de Justicia en Estados Unidos, que ella toma fuerza y comienza a ser implementada en el análisis de fusiones en particular y en los casos de libre competencia en general.

Una de las primeras críticas que recibió el documento de política de fusiones del Departamento de Justicia fue que los conceptos utilizados en general y la definición de mercado relevante en particular no podían ser

\footnotetext{
${ }^{4}$ Esto se conoce en la literatura como la falacia del celofán, debido a la definición de mercado relevante que consideró la Corte Suprema en Estados Unidos para el caso que involucraba a la empresa Du Pont.
} 
implementados "rigurosamente y a través del análisis de datos" (Stigler y Sherwin, 1985). Esta crítica, sin embargo, ha resultado equivocada, ya que el test del monopolista hipotético se puede implementar a través de un análisis de elasticidad de demanda o de pérdida crítica (Werden, 1998; Baumann y Godek, 1995; Harris y Simons, 1989).

La elasticidad de demanda crítica es la máxima elasticidad de demanda en un mercado relevante, antes de la fusión, tal que un monopolista hipotético aumentaría el precio en al menos un porcentaje mínimo establecido, típicamente $5 \%{ }^{5}$

La pérdida crítica, por otro lado, es la máxima reducción en la cantidad vendida que un monopolista hipotético podría soportar para sostener un aumento de precios.

Existen distintos tipos de evidencia que permiten con mayor o menor precisión implementar el test del monopolista hipotético:

\section{a) Reacción histórica de los consumidores a cambios de precios}

Cambios de precios en el pasado pueden reflejar cambios en los costos de toda la industria y no poder de mercado. Por esta razón la reacción de los consumidores a dichos cambios no necesariamente permite inferir su respuesta frente a un monopolista hipotético. Sin embargo, la evidencia histórica puede ser de utilidad en algunos casos. Lo importante para estos efectos es distinguir entre la evidencia de que los consumidores cambiaron su consumo a otros productos frente un aumento en el precio y la evidencia de que los consumidores no se cambiaron. Si un número importante de consumidores se cambió de un producto A a otro producto B debido a un aumento pequeño pero significativo en el precio del producto A, ello sería evidencia de que ambos productos pertenecen al mismo mercado relevante. La posibilidad de que el aumento en el precio del bien A sea producto de un aumento en los costos sugiere que incluso más consumidores se cambiarían al producto B si el aumento en el precio de A no tuviera justificación de costos y fuera el resultado del ejercicio de poder de mercado o fuera un aumento de mayor duración.

Por otro lado, el hecho de que los consumidores no se cambiaron del producto $\mathrm{A}$ al producto $\mathrm{B}$ como respuesta a un aumento pequeño pero significativo en el precio relativo de A, no necesariamente constituye evidencia de que ambos productos pertenecen al mismo mercado relevante, ya que es más probable que los consumidores se cambien al producto B si el

\footnotetext{
${ }^{5}$ En Nueva Zelanda se considera un aumento entre 5 y $10 \%$.
} 
aumento en el precio no tuviera justificación de costos o fuera de larga duración en el tiempo.

En el caso extremo, esto es, cuando las empresas que venden un producto han estado involucradas en el pasado en actividades colusivas en un mercado, no hay razón para dudar que el mercado relevante está automáticamente bien definido y consiste simplemente en el producto o servicio respecto al cual hubo acuerdos colusivos.

De igual forma hay regulaciones gubernamentales, como las bandas de precios, que permiten definir automáticamente un mercado relevante.

\section{b) Evidencia econométrica}

Existen varios tipos de evidencia econométrica que pueden ser utilizados para responder al test del monopolista hipotético. Un primer tipo de análisis consiste en utilizar experimentos naturales. Este tipo de análisis requiere información sobre precios, número de competidores y los factores que influyen en el precio de los bienes para consumidores finales.

Un segundo tipo de análisis estima directamente elasticidades de demanda y para ello se requiere información que varíe en el tiempo respecto a precio y cantidades. El parámetro crítico para la definición de mercado relevante es la elasticidad precio cruzada, que mide el cambio porcentual en la cantidad demandada de un bien en respuesta a un pequeño cambio porcentual en el precio de otro bien, dejando todo lo demás constante. Una elasticidad cruzada positiva y considerable en magnitud es evidencia de que dos bienes son sustitutos cercanos.

Un tercer tipo de análisis econométrico consiste en estimar la elasticidad residual de cada una de las empresas que se fusionan para posteriormente obtener la elasticidad residual de la empresa fusionada (Baker y Bresnahan, 1985, 1988; Sheffman y Spiller, 1987). Esta elasticidad mide la demanda que enfrenta una empresa una vez que la reacción de todas las otras empresas en el mercado es tomada en cuenta. Una elasticidad residual baja refleja poder de mercado de una empresa, ya que al aumentar el precio la mayoría de los consumidores seguirían comprando de dicha empresa en vez de cambiarse a otras.

Un cuarto tipo de análisis lo constituyen los tests de correlación (Stigler y Sherwin, 1985) y diferencial de precios (Horowitz, 1981; Huettner, 2002). La intuición detrás de estos tests es que si dos productos pertenecen al mismo mercado relevante sus precios deberían moverse de la misma manera en el tiempo. De igual forma, el diferencial de precios en el largo plazo de dos productos que son sustitutos muy cercanos debería tender a cero. 
Por esta razón, una correlación alta de precios entre dos productos o un diferencial de precios de largo plazo cercano a cero entre ellos constituiría evidencia de que son altamente sustitutos. Sin embargo, una alta correlación puede ser espuria, producto de shocks de costos o de demanda que son comunes a dos productos que no pertenecen al mismo mercado relevante. Si bien existen técnicas econométricas que pueden eliminar una correlación espuria en la estimación, el estándar es utilizar estos tests sólo para descartar que dos productos pertenezcan a un mismo mercado relevante.

En general, la evidencia econométrica constituye la mejor evidencia posible en la definición de un mercado, y la principal restricción en su implementación se encuentra en la disponibilidad de información. Ésta es una limitación particularmente importante en Chile que podría verse reducida si se establece en el Tribunal de la Libre Competencia, como sucede en varios países desarrollados, una etapa previa de recolección de información (discovery) en la que incluso se obliga a las empresas a entregar datos bajo condiciones estrictas de confidencialidad por parte de quienes utilizarán esos datos.

\section{c) Declaraciones de consumidores tipo}

Si el objetivo es averiguar qué haría un consumidor en un caso hipotético, una manera de hacerlo es preguntarle directamente al consumidor. Para estos efectos, en algunas cortes de otros países se interroga a los consumidores. Ya que la información entregada por los consumidores puede no ser confiable, se requiere preguntar a una muestra representativa de un grupo grande de consumidores por cada tipo de consumidores.

\section{d) Encuestas}

El objetivo de una encuesta es el mismo que el de interrogar directamente a los consumidores; sin embargo, sus resultados pueden ser mucho más confiables si se realiza utilizando una buena metodología que contemple muestras aleatorias, preguntas bien diseñadas y posibles sesgos de selección.

Una encuesta bien diseñada y realizada puede revelar las preferencias de los consumidores, sus patrones de consumo y su disposición a sustituir entre distintos bienes frente a cambios en sus precios relativos. 


\section{Empresas participantes, participaciones de mercado y concentración}

En esta etapa del análisis se debe determinar, en primer lugar, cuáles son las empresas que participan en el mercado relevante. Posteriormente, es necesario calcular la participación de mercado de cada una de esas empresas, para finalmente medir el grado de concentración en el mercado relevante y evaluar el riesgo que presenta la fusión.

\section{a) Empresas que participan en el mercado relevante}

Una vez definido el mercado relevante, es necesario identificar a las empresas que participan en dicho mercado. Para esto hay que identificar obviamente a las empresas que actualmente producen o venden sus servicios o productos en el mercado relevante. Sin embargo, es importante identificar también a las empresas que, si bien no producen ni venden sus servicios o productos en el mercado, empezarían a hacerlo rápidamente frente a un aumento significativo y no transitorio en los precios por parte del monopolista hipotético que ya se mencionó. Estas respuestas de la oferta ante un aumento de precios no transitorio pueden realizarse, por ejemplo, cambiando o extendiendo el uso de algunos activos de las empresas hacia la producción en el mercado relevante.

Empresas en condiciones de responder de esta forma, sin necesidad de incurrir en costos hundidos de entrada o salida, son denominadas en la literatura "entrantes no comprometidos" (uncommitted entrants) en el mercado relevante y deben ser consideradas como participantes en el mercado. En esta etapa del análisis no se deben considerar empresas que para poder competir en el mercado relevante tienen que incurrir en costos hundidos tanto en la producción como en la distribución del producto relevante ${ }^{6}$, ya que ello involucra más tiempo para poder participar en el mercado y los convierte en nuevos entrantes.

En este análisis se debe considerar particularmente el tiempo que se requiere para que ocurra el cambio en la oferta, las restricciones técnicas de cambiar la producción de un mercado a otro y sus costos asociados, los esfuerzos de marketing y los gastos involucrados en establecer una reputación creíble como proveedor en el mercado, el grado de inversiones tangibles e intangibles involucradas y el grado en el cual una respuesta de oferta ha ocurrido en el pasado en el mismo mercado.

${ }^{6}$ Por ejemplo, requerimientos de bodega, una red de distribución, costos de marketing, contratación de vendedores, obtención de permisos legales, etc. 


\section{b) Participaciones de mercado}

Las participaciones de mercado son relevantes cuando la principal preocupación se refiere a acciones unilaterales por parte de una empresa para aumentar los precios o actuar en contra de empresas rivales.

Una fusión que involucre a productores de bienes que son sustitutos cercanos puede relajar las fuerzas competitivas sobre cada producto al eliminar al principal sustituto. Esta posibilidad depende de la sustitución de demanda entre los dos bienes más que de la posible cooperación entre los distintos oferentes del bien. Si la sustitutividad entre productos es suficientemente alta, una fusión internaliza la sustitución que realizan los consumidores desde el bien más caro hacia el más barato. De esta forma, se reduce uno de los castigos de mercado que sufre una empresa que aumenta artificialmente los precios.

Las participaciones de mercado para cada uno de los participantes se calculan en base al total de ventas, producción o capacidad en el mercado relevante, por lo cual son expresadas en pesos o en unidades físicas ${ }^{7}$. Idealmente, para el cálculo de las participaciones de mercado se deberían incluir las ventas que tendría o la capacidad que utilizaría cada empresa en el mercado relevante en respuesta a un aumento pequeño pero significativo en el precio.

La práctica es utilizar datos anuales para medir la participación de mercado de las empresas participantes, pero en mercados con ventas infrecuentes o con demasiada incertidumbre en la demanda puede ser razonable utilizar períodos más largos.

\section{c) Concentración}

Al reducir el número de empresas en un mercado, una fusión aumenta la probabilidad de que las empresas que quedan en el mercado puedan comportarse, unilateralmente o cooperativamente, en forma no competitiva.

Hay algunos factores que influyen en el resultado final después de una fusión y por lo tanto son importantes en predecir la probabilidad de que existan o no efectos anticompetitivos a partir de una fusión en particular. La concentración en el mercado relevante y las participaciones de las empresas que se fusionan son dos factores importantes que deben ser considerados. Un tercer factor, que será discutido en la próxima sección, se refiere a las condiciones de entrada en el mercado relevante.

${ }^{7}$ En el caso de mercados de recursos naturales no renovables, como el cobre, puede ser útil emplear reservas. 
Si bien un alto grado de concentración no es suficiente para concluir que hay poder de mercado, sí constituye una de las condiciones necesarias para el ejercicio de poder de mercado. Por esta razón, es muy poco probable que una fusión genere o aumente poder de mercado si como resultado de ella el mercado relevante no termina siendo un mercado altamente concentrado.

El grado de concentración en un mercado relevante es importante cuando se está evaluando una fusión que tiene como efecto potencial mejorar y facilitar la coordinación entre empresas y/o el comportamiento estratégico concertado respecto a empresas rivales.

El comportamiento estratégico involucra esfuerzos para disminuir el grado de competitividad de empresas rivales, con consecuencias finales que son adversas para los consumidores ${ }^{8}$.

La concentración en un mercado es una función del número de empresas participantes en el mercado y sus respectivas participaciones de mercado. Una medida estándar de concentración es el Índice de HerfindahlHirschman $(H H I)$, el cual se calcula sumando las participaciones de mercado $(S)$ de cada empresa elevada al cuadrado:

$$
H H I=\sum_{i} S_{i}^{2}
$$

Este índice nace de modelos teóricos de oligopolio y tiene como ventaja respecto a otras alternativas ${ }^{9}$ el que utiliza información de todas las empresas participantes en el mercado y refleja su distribución. Además le otorga un mayor peso a las participaciones de mercado de las empresas más grandes. El $\mathrm{HHI}$ puede tomar valores entre 1 y un número pequeño cercano a 0 , mientras mayor es el valor del HHI, mayor es la concentración de empresas en el mercado.

Con el objeto de implementar una política de evaluación de fusiones, es útil dividir los mercados en tres tipos: no concentrados, moderadamente concentrados y altamente concentrados. Si bien cualquier división es arbitraria, se propone la siguiente separación:

${ }^{8}$ En la literatura económica especializada hay numerosos modelos que permiten explicar el foreclosure, esto es, el aumento artificial de los costos de los rivales, y el bundling, esto es, conductas anticompetitivas que no involucran directamente aumento de precios a consumidores en forma cooperativa o unilateral.

${ }^{9}$ En Australia y Nueva Zelanda se utiliza el índice de concentración C4, entre otras razones, porque el gobierno lo publica regularmente para todas las industrias. Sin embargo, el índice C4 utiliza sólo la información de unas pocas empresas, las más grandes, y descarta información importante respecto al tamaño relativo de las empresas más pequeñas y a la distribución del tamaño de empresas en el mercado. 


\begin{tabular}{|ll|}
\hline Mercado & HHI \\
\hline No concentrado & Menor que 0,2 \\
Moderadamente concentrado & Entre 0,2 y 0,25 \\
Altamente concentrado & Mayor que 0,25 \\
\hline
\end{tabular}

Esta separación arbitraria que se propone es menos estricta que la establecida en la política de fusiones de Estados Unidos ${ }^{10}$ y está basada en la evidencia empírica de fusiones aprobadas y rechazadas por la FTC y el DOJ en los últimos 15 años.

En el caso de fusiones que involucran un mercado no concentrado después de la fusión, es altamente probable que no haya efectos anticompetitivos y dicha fusión debiera aprobarse sin mayor análisis.

En el caso de fusiones que involucran un mercado moderadamente concentrado después de la fusión, dicha fusión debería aprobarse sin mayor análisis si el cambio en el $\mathrm{HHI}$ es menor que 0,02 y debería hacerse un análisis más detallado de los factores de entrada y las ganancias de eficiencia si el cambio en el $\mathrm{HHI}$ es mayor que 0,02.

Finalmente, en el caso de fusiones que involucran un mercado altamente concentrado, si el aumento en el HHI es menor que 0,0125 la fusión debiera aprobarse sin análisis adicionales y debiera hacerse un análisis de los otros factores si el cambio es mayor que 0,0125.

\section{Entrada}

En general, es poco probable que una fusión genere o aumente el poder de mercado si entrar a competir en el mercado relevante es tan fácil y rápido que los participantes en el mercado, colectiva o unilateralmente, no pueden sostener en forma rentable un aumento de precios a niveles superiores a los que existían antes de la fusión. Para que esto ocurra se necesita que no haya barreras a la entrada y que los potenciales entrantes puedan entrar a competir en el mercado oportunamente en tiempo y magnitud.

\section{a) Barreras a la entrada}

Joe Bain (1956) define barreras a la entrada como factores que permiten que las empresas ya establecidas en un mercado obtengan utilidades

${ }^{10}$ La política de fusiones en Estados Unidos define como mercados no concentrados a los que tienen un $\mathrm{HHI}$ menor a 0,1, como moderadamente concentrados a los que tienen un $\mathrm{HHI}$ entre 0,1 y 0,18 , y como altamente concentrados a los que tienen un HHI superior a 0,18 . 
supranormales sin que exista la amenaza de entrada por parte de potenciales empresas competidoras. De esta forma, Bain identifica tres posibles barreras a la entrada que pueden ser la fuente de utilidades supranormales para una empresa: ventajas de costo por parte de empresas ya establecidas en un mercado $^{11}$, diferenciación de productos ${ }^{12}$ y economías de escala ${ }^{13}$.

George Stigler (1983), por otro lado, define barreras a la entrada como los costos adicionales de largo plazo en que debe incurrir un entrante respecto a los costos de largo plazo que enfrenta una empresa que ya está en el mercado (incumbente). Las barreras a la entrada se basarían en este caso en asimetrías de costos entre las empresas establecidas y las potenciales entrantes. En ese sentido la definición de Stigler es consistente con las primeras dos barreras a la entrada identificadas por Bain (ventajas de costo y diferenciación de producto), pero no con la tercera (economías de escala).

Una definición concordante con la de Stigler es la de Von Weizsacker (1980), que define barreras a la entrada como los costos que enfrentan las firmas que deseen entrar a la industria, pero que no enfrentan aquellas que ya están establecidas en esa industria. En general, existe cierto grado de consenso entre los economistas en aceptar una definición de barreras a la entrada más cercana a la definición de Stigler (ver, por ejemplo, Jacquemin, 1987, y Waterson, 1984). Así, las posibles fuentes de barreras a la entrada pueden ser clasificadas en tres tipos: barreras legales o regulatorias, costos hundidos y tecnologías en uso.

\section{b) Potenciales entrantes}

En el análisis de barreras a la entrada y la potencial entrada de nuevos competidores hay que considerar particularmente a las empresas que

${ }^{11}$ En este caso una empresa entrante tiene costos de producción más altos en todos los niveles que una empresa establecida. Una empresa incumbente puede tener tecnologías más eficientes de producción, las cuales puede haber adquirido a través de la experiencia o la investigación y desarrollo. Puede incluso haber acumulado capital que reduce su costo de producción o haber impedido el acceso a un insumo esencial a sus potenciales competidores (a través de contratos con los proveedores, por ejemplo).

${ }^{12}$ Empresas ya establecidas en un mercado pueden tener patentes de innovaciones para un producto, pueden haber encontrado un nicho de mercado o pueden contar con la lealtad de sus consumidores. En este último caso, los consumidores prefieren consumir marcas conocidas de las empresas ya establecidas en vez de marcas desconocidas de empresas entrantes. Por esta razón una empresa entrante debe destinar un mayor esfuerzo a ventas, marketing y publicidad (por peso vendido) que una empresa ya establecida.

${ }^{13}$ Si la escala mínima eficiente de producción representa una fracción importante de la demanda en un mercado, puede existir en ese mercado un número pequeño de empresas que obtengan utilidades supranormales sin que haya entrada de nuevos competidores. 
deben incurrir en algún tipo de costo hundido para entrar al mercado ${ }^{14}$. Estos potenciales entrantes evalúan entonces la rentabilidad de entrar basados en el largo plazo y se denominan "entrantes comprometidos" (committed entrants).

Un entrante comprometido evalúa la rentabilidad de entrar a un mercado en el largo plazo, considerando para esto el precio en el mercado después de haber entrado, ya que éste es el precio que recibirá por sus productos. Es por ello que en su decisión de entrada debe considerar el efecto que tiene en el precio de mercado un aumento en la oferta y la reacción de las empresas que ya se encuentran en el mercado, todo lo cual reduce el precio de largo plazo post-entrada (el cual será más bajo que el precio pre-entrada que reflejaba algún grado de poder de mercado de los incumbentes).

Para que la potencial entrada de una empresa tenga como efecto impedir que las empresas establecidas puedan ejercer poder de mercado en el mercado relevante, esta entrada debe ser probable ${ }^{15}$, oportuna ${ }^{16}$ y suficiente en magnitud ${ }^{17}$.

En un mercado en el cual la entrada de un nuevo competidor es probable, oportuna y suficiente frente a un aumento pequeño pero significativo en los precios después de una fusión, dicha fusión no es motivo de preocupación respecto a efectos anticompetitivos y debe aprobarse sin mayor análisis.

Para evaluar si es probable que un nuevo competidor pueda entrar a competir en un mercado relevante en forma oportuna y suficiente para restaurar la competencia, se deben considerar todas las acciones que una empresa tiene que emprender para producir y vender en dicho mercado. Para estos efectos hay que considerar todos los procesos de planificación, diseño, aprobaciones, permisos, construcción, publicidad, distribución, etc.

${ }^{14}$ La razón para ello es estrictamente metodológica, ya que los potenciales entrantes que no incurren en costos hundidos están incluidos dentro de los participantes en el mercado relevante.

${ }^{15} \mathrm{La}$ probabilidad de entrada depende fundamentalmente de que sea rentable hacerlo, lo cual debe evaluarse, para una empresa que incurre en costos hundidos para entrar, a precios competitivos y no a los precios antes de la entrada.

${ }^{16}$ Para que los efectos de la entrada de un competidor reduzcan el poder de mercado de las empresas establecidas, es necesario que el impacto en los precios en el mercado relevante ocurra relativamente rápido.

${ }^{17} \mathrm{Si}$ bien la entrada de un competidor puede ser probable y oportuna, para que efectivamente tenga efectos en la competencia en el mercado relevante debe ser, además, suficiente en magnitud para devolver los precios a niveles competitivos. Esto puede ocurrir por la entrada de varios nuevos competidores o por la entrada de un nuevo competidor con una escala suficiente. 
El análisis consiste en evaluar tres aspectos de la potencial entrada de un nuevo competidor al mercado.

En primer lugar, se debe evaluar si un entrante puede lograr un impacto significativo en el mercado en un período razonable, generalmente uno a dos años.

En segundo lugar se debe evaluar si la entrada al mercado será rentable y, por lo tanto, una respuesta probable a fusión que tiene efectos anticompetitivos. Una empresa que considere entrar al mercado debe evaluar la rentabilidad de esta acción bajo el supuesto de una participación de largo plazo en el mercado, ya que hay costos hundidos involucrados. La entrada de un nuevo competidor que logre contrarrestar los efectos competitivos de una fusión necesariamente llevará el precio de mercado a los niveles previos a la fusión. Por esta razón, la rentabilidad de un potencial entrante debe evaluarse a los precios existentes en el mercado antes de la fusión (al menos no mayores que los pre-fusión).

Lo anterior puede aparecer como una contradicción, ya que si es rentable entrar al mercado a los precios que había antes de la fusión, ¿por qué no entraron antes nuevos competidores? La respuesta a esta contradicción aparente es que el mercado después de la fusión es distinto al de antes de la fusión. Si la fusión generó poder de mercado, el precio debe ser mayor y la cantidad menor que antes de la fusión. El hecho de que la cantidad en equilibrio en el mercado sea menor es precisamente lo que genera oportunidades de nuevas ventas para un entrante a un nivel mayor que el que estaba disponible antes de la fusión y eso hace que la entrada después de la fusión sea rentable aunque no lo haya sido antes de la fusión (Salop, 1986).

Un elemento de particular consideración en este análisis lo constituye la escala mínima de viabilidad (EMV), que consiste en el nivel mínimo de ventas promedio, a los precios previos a la fusión, que un entrante debe tener para que sea rentable entrar y permanecer en el mercado.

La EMV depende de los ingresos esperados y los costos asociados, incluyendo una tasa de retorno sobre el capital invertido. Por ello, la EMV es relativamente alta cuando los costos fijos de entrada son altos, cuando los costos de entrada son principalmente costos hundidos y cuando los costos marginales de producción son altos a niveles bajos de producción. En este sentido, la probabilidad de entrada en el mercado es muy baja si la EMV es mayor que el volumen de ventas disponible para potenciales entrantes. Dicho volumen disponible depende principalmente de la reducción en las ventas que ocurriría por los efectos anticompetitivos de la fusión y de la habilidad del entrante para capturar una fracción del crecimiento espe- 
rado de la demanda en el mercado. Por otro lado, el volumen de ventas disponible para el entrante puede ser bajo porque el entrante está excluido de una fracción importante del mercado (por integración vertical del incumbente por ejemplo) o porque el incumbente expande sus ventas en forma importante utilizando inversiones previas irreversibles en exceso de capacidad de producción.

En las políticas de fusiones de varios países se compara la EMV de un entrante, medida como fracción de las ventas anuales de la industria, con una estimación de las oportunidades de ventas que tiene un entrante después de ocurrida la fusión. Como criterio se usa que si la EMV es superior en $5 \%$ al total de las ventas en el mercado es poco probable que la entrada sea rentable. Obviamente que este criterio debe considerar algunos factores adicionales que pueden modificar la regla del $5 \%$, como el crecimiento esperado de la demanda en el mercado, los niveles de integración vertical de los incumbentes y la respuesta de los incumbentes a la entrada de un nuevo competidor (Scheffman y Spiller, 1992; Werden y Froeb, 1998).

En tercer lugar, se debe evaluar si la entrada probable y oportuna de un nuevo competidor será suficiente para restaurar un equilibrio competitivo en un mercado que pasó a ser menos competitivo después de una fusión. Este efecto se puede lograr por la entrada de un solo competidor con escala suficiente o de varios pequeños competidores que en conjunto logran dicha escala ${ }^{18}$.

En todo el análisis respecto a la potencial entrada en el mercado por parte de "entrantes comprometidos" se deben considerar especialmente las conductas estratégicas que pueden dificultar o incluso impedir la entrada ${ }^{19}$.

Existen varios modelos en la literatura económica cuya pregunta central es la probabilidad de entrada cuando hay costos hundidos. Dichos modelos consideran distintas respuestas de empresas incumbentes frente a la entrada de un nuevo competidor. Si se espera, por ejemplo, que los incumbentes reaccionen rápidamente bajando sus precios como respuesta a la entrada de un nuevo competidor, la entrada puede ser impedida incluso si los precios antes de la entrada son mayores que los precios de un mercado competitivo (Salop, 1979; Ordover y Wall, 1988). Esto ocurre porque el potencial entrante, reconociendo el nivel de competencia post-entrada, no es-

${ }^{18}$ Un ejercicio simple de implementar para tener una idea de la magnitud de entrada requerida para restablecer un equilibrio competitivo es el propuesto por Ricardo Sanhueza, que consiste en calcular la participación de mercado que tendría que lograr el entrante para restablecer el $\mathrm{HHI}$ existente antes de la fusión.

${ }^{19}$ Inversión en exceso de capacidad, precios predatorios, aumento de "costos de cambio" y "bundling”. 
pera obtener un margen de ganancias (ingresos menos costos variables) que permita cubrir sus costos hundidos.

\section{c) Mercados contestables}

Si los costos fijos de entrada no representan costos hundidos y un entrante tiene costos variables que son equivalentes a los de un incumbente, el mercado es "contestable" o "desafiable" y funciona en forma competitiva independiente del grado de concentración (Baumol, Panzar y Willig, 1988).

Las consideraciones estratégicas no son importantes cuando la entrada es de "entrantes no comprometidos", ya que en este caso se espera que una empresa que tiene una oportunidad de corto plazo de obtener utilidades desviando producción hacia un mercado específico efectivamente lo haga, incluso si dicha oportunidad desapareciera después de que entre en el mercado. Esta última estrategia se denomina hit and run y es la que se encuentra detrás del resultado de Baumol, Panzar y Willig.

Es importante aclarar bien el concepto de "mercado contestable" y los supuestos en que se basa, ya que en muchas presentaciones al Tribunal de la Libre Competencia se utiliza este resultado pero en forma incompleta o equivocada.

La teoría de mercados contestables o desafiables, elaborada por Baumol, Panzar y Willig (1982), establece que un mercado es desafiable (o contestable) si se satisfacen tres condiciones. La primera es que las empresas entrantes no enfrenten ninguna desventaja respecto a las empresas que ya están en el mercado (incumbentes). Esto implica que un entrante tiene el mismo acceso a la tecnología de producción, precios de insumos e información respecto a la demanda, es decir, no hay barreras a la entrada. La segunda condición es que no existan costos hundidos, es decir, que todos los costos asociados con la entrada al mercado sean completamente recuperables. Esto implica que no hay costos de salida. La tercera condición es que el tiempo que toma entrar sea menor que el tiempo que se demoran las empresas incumbentes en ajustar los precios.

El resultado central de la teoría de mercados contestables es que si se cumplen las tres condiciones anteriores, el equilibrio en el mercado es un equilibrio competitivo. La razón de este resultado es que si las empresas incumbentes suben el precio a niveles superiores al equilibrio competitivo, una nueva empresa puede entrar en el mercado, cobrar un precio menor, obtener utilidades positivas y salir del mercado antes de que las empresas 
incumbentes respondan ajustando sus precios. Esta estrategia por parte de las potenciales entrantes se denomina una estrategia de hit and run, y tiene como efecto impedir que un incumbente pueda cobrar un precio superior al competitivo. En este sentido, la competencia potencial disciplina a las empresas incumbentes, las cuales en equilibrio se comportan competitivamente sin que ocurra entrada.

Las tres condiciones señaladas previamente son de crucial importancia para obtener el resultado que establece la teoría de mercados desafiables. Los mismos autores de la teoría señalan que la existencia de costos hundidos, dependiendo de su magnitud, puede eliminar la posibilidad de que un entrante obtenga utilidades positivas antes de que los incumbentes respondan (ver también Gelman y Salop, 1983, y Stiglitz, 1987) ${ }^{20}$. De igual forma, si el tiempo que toma entrar al mercado es mayor que el tiempo que les toma a los incumbentes ajustar su precio, el precio de equilibrio será superior al competitivo (Schwartz y Reynolds, 1983).

Por alguna extraña razón, generalmente se considera y se discute sólo una de las tres condiciones necesarias para que un mercado sea contestable: barreras a la entrada. Un análisis económico correcto exige discutir y analizar las tres condiciones y no sólo una, por lo que ése es el estándar que la Fiscalía Nacional Económica debería usar y el Tribunal de la Libre Competencia exigir cuando se discuta la desafiabilidad de un mercado ${ }^{21}$.

Finalmente, para concluir esta sección, es importante reconocer que es mucho más difícil cuantificar los efectos de las condiciones de entrada en un mercado que calcular concentración o participaciones de mercado. Sin embargo, eso no significa que conceptos como EMV u oportunidades de venta sean irrelevantes o inútiles. Por el contrario, permiten concentrar los esfuerzos de la investigación y de la búsqueda de información en los factores relevantes que determinan si la entrada comprometida de nuevos competidores es rentable en el mercado de post-entrada.

${ }^{20}$ En palabras del propio Baumol: "Donde los mercados contestables se metieron en problemas, y correctamente, fue cuando algunos abogados los usaron para decir que desafiabilidad implica que no puede haber poder de mercado. Nosotros nunca dijimos eso. Lo que dijimos es que la 'desafiabilidad' perfecta es un constructo teórico, el cual no es más realista que la competencia perfecta, pero que es más útil como guía para regulación” (en Krueger, 2001).

${ }^{21}$ Adicionalmente, hay quienes en Chile usan como evidencia para "demostrar" que un mercado es contestable el que haya habido entrada de nuevos competidores en el mercado. Este argumento es equivocado, ya que en un mercado contestable no hay entrada en equilibrio. 


\section{Eficiencias}

El objetivo de una política de competencia es proteger la libre competencia como un medio para lograr una eficiente asignación de recursos. El tema de fondo y el objetivo final es eficiencia y no competencia per se $\mathrm{s}^{22}$. La libre competencia permite obtener eficiencia asignativa, productiva y dinámica.

\section{a) Eficiencia asignativa}

La eficiencia asignativa se logra cuando los recursos son asignados al uso más valorado entre todos los usos posibles, es decir, el valor de un producto para los consumidores es igual en el margen al valor de los recursos utilizados para producirlo. El primer "teorema fundamental del bienestar” muestra que el funcionamiento de un mercado competitivo garantiza la asignación eficiente de los recursos.

\section{b) Eficiencia productiva}

La eficiencia productiva se logra cuando los bienes se producen al mínimo costo posible, es decir, cuando no se pueden organizar los recursos en una forma distinta tal que aumente la producción de un bien y no disminuya la producción de otro bien. Una fusión puede aumentar la eficiencia productiva a través de economías de escala, economías de ámbito y sinergias.

Las economías de escala existen cuando el costo unitario de producción disminuye al aumentar la escala de producción ${ }^{23}$.

Las economías de ámbito existen cuando es más barato producir dos o más bienes en forma conjunta en vez de separadamente, porque se usan insumos comunes por ejemplo.

Las sinergias permiten obtener ganancias en la eficiencia productiva al integrar el uso de distintos activos específicos.

\section{c) Eficiencia dinámica}

La eficiencia dinámica se logra al incentivar la innovación con el objeto de reducir costos y desarrollar nuevos y mejores bienes y servicios.

${ }^{22}$ Ver, por ejemplo, Summers (2001).

${ }^{23}$ Farrel y Shapiro (2001) consideran que hay que ser escéptico de las ganancias de economías de escala, ya que las empresas pueden aumentar su escala comprando más insumos o expandiendo su capacidad. 
En este sentido es importante considerar las condiciones bajo las cuales el conocimiento tecnológico se expande en el tiempo (a través de investigación y desarrollo, creatividad empresarial o learning by doing, por ejemplo).

Uno de los aspectos críticos de la eficiencia dinámica consiste en establecer mecanismos que les permitan a los inventores obtener retornos suficientes de sus invenciones para hacer rentable la actividad de investigación y desarrollo. Ello puede requerir en algunos casos establecer barreras a la entrada en algunos mercados (Demsetz, 1982).

Oliver Williamson (1969) fue el primero en señalar que al evaluar una fusión era necesario considerar también los efectos positivos en eficiencia que potencialmente se podían obtener. En un influyente artículo Williamson fue capaz de mostrar el difícil trade-off entre eficiencia y poder de mercado que debe tenerse en cuenta al evaluar una fusión.

La figura siguiente muestra la idea básica en la que se basa el análisis de Williamson:

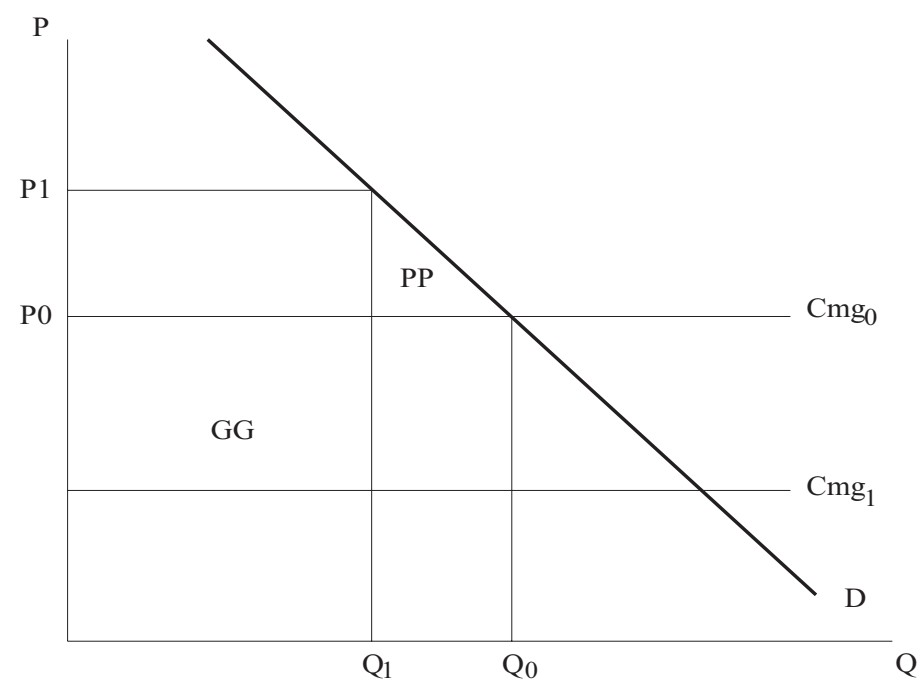

La figura muestra los equilibrios en un mercado antes y después de una fusión que simultáneamente genera poder de mercado y produce eficiencias. Las líneas horizontales $\mathrm{Cmg}_{0}$ y $\mathrm{Cmg}_{1}$ representan la curva de costo marginal de producir el bien antes y después de la fusión respectivamente. Antes de la fusión el mercado es perfectamente competitivo, por lo que en equilibrio se produce $\mathrm{Q}_{0}$ y el precio de venta es $\mathrm{P}_{0}$. Producto de la fusión, se 
genera poder de mercado, por lo que el precio aumenta a $\mathrm{P}_{1}$ y la cantidad se reduce a $\mathrm{Q}_{1}$. Como resultado de esta fusión hay dos efectos. El primero es una pérdida de bienestar, equivalente al área PP, producto del poder de mercado. El segundo es una ganancia de bienestar, equivalente al área GG, producto de las eficiencias generadas por la fusión.

De este simple análisis se desprende que puede existir un trade-off entre poder de mercado y ganancias de eficiencia, en el que potencialmente estas últimas pueden más que compensar las pérdidas generadas por lo primero. En un caso extremo, las ganancias de eficiencia pueden ser tan grandes que el precio post-fusión es menor, en cuyo caso la fusión es procompetitiva.

Posteriormente, la idea de Williamson fue recogida por Areeda y Turner en el influyente tratado legal de antitrust. En dicho tratado, señalan que las ganancias de eficiencia deben ser consideradas en forma importante por tres razones:

Primero, es poco probable que fusiones de empresas pequeñas e ineficientes generen poder de mercado; por lo demás, incluso esas fusiones podrían hacer más intensa la competencia. Segundo, aun en el caso en que una fusión reduzca la competencia en precios, el efecto negativo en bienestar puede ser más que compensado por el efecto positivo en bienestar de una mayor eficiencia. Tercero, impedir una fusión que genera grandes ineficiencias puede resultar inútil porque las empresas ineficientes probablemente desaparecerán producto de la competencia, con lo que el mercado terminará igualmente concentrado que en el caso en que se hubiera aprobado la fusión.

Las ganancias de eficiencia deben ser establecidas con evidencia clara y convincente. Adicionalmente, la fusión debe ser necesaria para lograr dichas eficiencias. En este sentido, lo relevante es establecer que las ganancias de eficiencia no se pueden obtener a través de alternativas que sean menos restrictivas (en particular, no provienen de reducir la cantidad producida ni la calidad). Esto es un aspecto importante y poco entendido por muchos. Una reducción en la cantidad producida probablemente tenga como resultado una reducción en los costos totales, pero esa reducción de costos no constituye una ganancia de eficiencia. De igual forma, al desaparecer la competencia entre dos empresas que se fusionan, la nueva empresa puede bajar los costos de captar nuevos clientes (publicidad, por ejemplo) o gastar menos en entregar servicios a sus clientes. Para la empresa fusionada ello constituye una reducción en sus costos, pero nuevamente esto no es una ganancia de eficiencia. El objetivo debe ser considerar si mejora la eficiencia asignativa o no. 
Las ganancias de eficiencia son difíciles de verificar y cuantificar, mayormente porque hay información asimétrica: las empresas que se fusionan tienen gran parte de la información y no la Fiscalía Nacional Económica o el Tribunal de la Libre Competencia. Por esta razón, el peso de la prueba debe caer en las empresas que se fusionan y las ganancias de eficiencia que se señalen no deberían ser consideradas si son generales, vagas o simplemente especulativas.

Una de las discusiones no resueltas se refiere a si las ganancias de eficiencia producto de una fusión deben ser traspasadas a los consumidores o no para ser consideradas. Una visión, a la cual adscribo, señala que todas las eficiencias deben considerarse porque lo que importa es el bienestar total, es decir, la suma de los excedentes del consumidor y el productor. Otra visión considera que el bienestar del consumidor es el que importa, en cuyo caso sólo interesan las eficiencias que se traspasan al consumidor a través de menores precios y mayor cantidad producida ${ }^{24}$.

\section{8. ¿Consulta obligatoria o voluntaria?}

Un último aspecto relevante en una política de evaluación de fusiones consiste en determinar si dos empresas que desean fusionarse están obligadas a solicitar una aprobación ex ante a los organismos que velan por la libre competencia o no.

En Chile, la ley no obliga a las empresas a obtener una aprobación previa a la fusión por parte del Tribunal de la Libre Competencia (TdLC). Sin embargo, el TdLC en el Auto Acordado N5/2004 estableció un procedimiento que incentiva las consultas ex ante al tribunal.

El procedimiento señala que cuando se ingrese una consulta sobre hechos o contratos ya ocurridos, la consulta será tratada bajo el procedimiento contencioso al haber cualquier oponente o demanda. En cambio, si la consulta se hace respecto a actos que no han ocurrido, no se puede posteriormente presentar una demanda o un requerimiento, por lo que los oponentes deben utilizar el procedimiento no contencioso.

En general, la principal razón para preferir consultas ex ante en el caso de fusiones es una de economía política: es más fácil y más probable impedir una fusión que posteriormente separar dos empresas ya fusionadas.

\footnotetext{
${ }^{24}$ Una de las principales contradicciones que genera la visión de sólo considerar el bienestar del consumidor es que la generación de monopsonios, por consistencia en la argumentación, debiera ser aprobada e incluso incentivada, a pesar de que la teoría económica muestra que inequívocamente se genera una distorsión en la asignación de los recursos.
} 
Esto es particularmente importante en un país como Chile, donde un juicio para separar una empresa fusionada puede demorar muchos años. Por otro lado, exigir obligatoriedad de consulta para todas las fusiones puede generar un exceso de causas para el tribunal, lo cual no sólo implica un gasto importante en recursos para evaluar cada potencial fusión sino que además es innecesario, ya que hay muchas fusiones que tienen escasas probabilidades de generar poder de mercado. Por ejemplo, una fusión entre dos empresas que tienen $2 \%$ del mercado relevante cada una es altamente improbable que tenga algún efecto negativo en la competencia.

El procedimiento establecido por el TdLC es un mecanismo razonable, ya que pone los incentivos correctos para que una fusión que tenga más probabilidades de ser cuestionada sea presentada en consulta al tribunal. Sin embargo, sería importante establecer que una vez hecha la consulta al Tribunal ésta no puede retirarse. De otra forma se abre la posibilidad de que las empresas que consultan al Tribunal actúen en forma estratégica.

\section{Conclusiones y comentarios finales}

Con el objeto de sistematizar el análisis requerido para evaluar si una fusión puede tener efectos anticompetitivos o no, se debería avanzar hacia el establecimiento de una política explícita de evaluación de fusiones. Esto no sólo facilitaría el trabajo de la Fiscalía Nacional Económica y del Tribunal de la Libre Competencia, sino que además reduciría la incertidumbre regulatoria que enfrentan las empresas y reduciría los costos de cumplimiento.

En este trabajo se propone una política de fusiones explícita para Chile, que siga los principios de las políticas de fusiones utilizadas por países desarrollados. Con este objeto, se establece un marco analítico de cuatro etapas, consistentes en (1) definir el mercado relevante; (2) evaluar la concentración en dicho mercado y los potenciales efectos anticompetitivos de la fusión; (3) evaluar las condiciones de entrada, y (4) evaluar los potenciales efectos de eficiencia producto de la fusión.

Es importante señalar que al implementar una política de evaluación de fusiones como la que se propone en este trabajo, consistente en realizar un análisis a través de una serie de etapas en cada una de las cuales la respuesta debe ser específica en un sentido para considerar que la fusión es anticompetitiva, y donde la evaluación y las respuestas que se obtienen en cada etapa son inciertas en algún grado, es fácil llegar a conclusiones equivocadas si se tratan las respuestas de cada etapa por separado y se da una etapa por concluida antes de moverse a la etapa siguiente. El análisis debe ser completo y riguroso, de otra forma los resultados pueden ser equivocados. 
Por último, dos ámbitos complementarios que deben ser considerados en el análisis. El primero atañe al peso de la prueba. En principio, el peso de la prueba respecto a los potenciales efectos anticompetitivos de una fusión debería recaer en la Fiscalía Nacional Económica o en quien se oponga a la fusión, y respecto a las ganancias de eficiencia que entrega la fusión, el peso de la prueba debería caer en las empresas que quieren fusionarse.

El segundo ámbito se refiere a restricciones que disminuyan los riesgos de que una fusión tenga efectos anticompetitivos. Una solución posible al trade-off entre ganancias de eficiencia y poder de mercado consiste en aprobar una fusión sujeta a ciertas condiciones que, por un lado, maximicen la probabilidad de que se realicen las ganancias de eficiencia y, por otro lado, minimicen las probabilidades de que se genere poder de mercado. Hay fusiones, por ejemplo, que pueden generar riesgos competitivos sólo en algunos mercados geográficos o en algunos mercados de producto dentro de los múltiples productos que venden las empresas involucradas. En estos casos existen medidas de mitigación, como por ejemplo venta de algunos activos, que permiten que la fusión se lleve a cabo sin riesgos. Si bien el Tribunal de la Libre Competencia ha implementado esta solución en algunas oportunidades, no existe en Chile un mecanismo que permita que las empresas que se fusionan ofrezcan y negocien con la Fiscalía Nacional Económica este tipo de medidas, de tal forma de llegar a un acuerdo que les garantice que la fusión no será objetada. Este mecanismo existe y se utiliza exitosamente en otros países y me parece importante considerar su implementación en Chile, haciendo para ello los cambios legales que se requieran.

Para concluir, quisiera mencionar que a pesar del gran número de fusiones que se concretan cada año, no existe evidencia empírica robusta en la literatura respecto al efecto que tienen las fusiones. En parte, esto se debe a la falta de datos que permitan un análisis econométrico riguroso. Sin embargo, la mayor dificultad radica en poder distinguir si los cambios en precios que se observan se deben a la fusión o a otros cambios en la industria. Adicionalmente, los efectos de una fusión no se limitan a cambios en precios sino que potencialmente también a cambios en la calidad del bien, el servicio ofrecido o innovación.

Para complicar las cosas aún más, si la política de libre competencia que implementa un país es la correcta, las fusiones que podrían haber tenido efectos anticompetitivos fueron bloqueadas por los organismos de defensa de la libre competencia o nunca fueron presentadas para su evaluación por las partes interesadas, por lo que es imposible observar si los resultados negativos esperados se concretaron o no. 
A pesar de estas limitaciones, existen estudios en la literatura que han intentado evaluar los efectos de una fusión. La evidencia de estos estudios no es concluyente ni respecto al efecto en precios y calidad de servicio, como tampoco respecto a las ganancias de eficiencia (un buen resumen de estos estudios se encuentra en Pautler, 2003, y Whinston, $2006)^{25}$.

Si bien no podemos sacar conclusiones generales de la evidencia en la literatura respecto al impacto de las fusiones aprobadas, en el caso de Chile, y con el objeto de mejorar los procedimientos y las futuras evaluaciones de fusiones (ver Kovacic, 2001), la Fiscalía Nacional Económica debiera realizar en forma sistemática una evaluación ex post de sus decisiones y de los resultados obtenidos.

\section{REFERENCIAS}

Adelman, Morris A. (1959): “Economic Aspects of the Bethlehem Opinion”. En Virginia Law Review, 45.

Australian Competition and Consumer Commission (1996): "Merger Guidelines".

Bain, Joe S. (1956): Barriers to New Competition. Harvard University Press.

Baker, J. B. y T. F. Bresnahan (1985): "The Gains from Merger or Collusion in Product Differentiated Industries”. En Journal of Industrial Economics, 33.

(1988): “Estimating the Residual Demand Curve Facing a Single Firm”. En International Journal of Industrial Organization, 6.

Baumann, Michael G. y Paul E. Godek (1995): “Could and Would Understood: Critical Elasticities and the Merger Guidelines”. En Antitrust Bulletin, 40.

Baumol, William J., John C. Panzar y Robert D. Willig (1988): Contestable Markets and the Theory of Industry Structure. Harcourt Brace Jovanovich Academic Press.

Bureau of Competition Policy (1991): "Merger Enforcement Guidelines”. Canada.

Commerce Commission (1996): “Business Acquisition Guidelines”. New Zealand.

Demsetz, H. (1982): “Barriers to Entry”. En American Economic Review, 47.

European Competition Commission (2002): "Commission Notice on the Appraisal of Horizontal Mergers”. Brussels.

Farrel, J. y C. Shapiro (2001): "Scale Economies and Synergies in Horizontal Merger Analysis”. En Antitrust Law Journal, 685.

Fisher, Franklin M. (1987): "Horizontal Mergers: Triage and Treatment”. En Journal of Economic Perspectives, 23.

Gelman, Judith R. y Steven C. Salop (1983): “Judo Economics: Capacity Limitation and Coupon Competition”. En Bell Journal of Economics, 14.

${ }^{25}$ Whinston (2006) señala que "Lamentablemente, la literatura económica contiene muy pocos análisis de este tipo” (p. 115). En el caso de las ganancias de eficiencia las cosas no son muy distintas y "hasta ahora, la evidencia respecto a los efectos en eficiencia de las fusiones horizontales sirve poco de guía” (p. 131). 
Harris, Barry C. y Joseph J. Simons (1989): "Focusing Market Definition: How Much Substitution Is Enough?”. En Research in Law and Economics, 207.

Horowitz, Ira (1981): "Market Definition in Antitrust Analysis: A Regression Based Approach". En Southern Economic Journal, 48.

Hoseken, Daniel, Daniel O’Brien, David Scheffman y Michael Vita (2002): “Demand System Estimation and its Application to Horizontal Merger Analysis”. Federal Trade Commission Bureau of Economics, Working Paper $N^{\circ} 246$.

Huettner, David A. (2002): "Product Market Definition in Antitrust Cases when Products Are Close Substitutes or Close Complements”. En Antitrust Bulletin 47(1).

Jaquemin, Alexis (1987): The New Industrial Organization. MIT University Press.

Kovacic, William E. (2001): "Evaluating Antitrust Experiments: Using Ex Post Assessments of Government Enforcement Decisions to Inform Competition Policy”. En George Mason Law Review, 9.

Krueger, A. (2001): “An Interview with William Baumol”. En Journal of Economic Perspectives, 15 (3).

Office of Fair Trading, United Kingdom (1999): “The Competition Act of 1998: Market Definition”. United Kingdom.

Ordover, Jansuz A. y Daniel M. Wall (1988): “A Practical Guide to the Economics of New Entry". En Antitrust, 2.

Oster, Sharon M. (1999): Modern Competitive Analysis. Oxford University Press.

Pautler, P. (2003): “Evidence on Mergers and Acquisitions”. En Antitrust Bulletin, 48.

Posner, Richard A. (1976): Antitrust Law: An Economic Perspective. University of Chicago Press, primera edición.

Salop, Steven C. (1979): “Strategic Entry Deterrence”. En American Economic Review, 69 (2). (1986): “Measuring Ease of Entry”. En Antitrust Bulletin, 31.

Scheffman, David T. y Pablo T. Spiller (1987): “Geographic Market Definition under the U.S. Department of Justice Merger Guidelines”. En Journal of Law and Economics, 30.

(1992): “Buyer's Strategies, Entry Barriers, and Competition”. En Economic Inquiry, 30.

Schwartz, Marius y Robert J. Reynolds (1983): "Contestable Markets: An Uprising in the Theory of Industry Structure: Comment”. En American Economic Review 73(3).

Stigler, George (1950): “Monopoly and Oligopoly by Merger”. En American Economic Review, 40.

(1971): “The Theory of Economic Regulation”. En Bell Journal of Economics and Management Science, 2. (1983): The Organization of Industry. University of Chicago Press.

Stigler, George y Robert Sherwin (1985): “The Extent of the Market”. En Journal of Law and Economics, 28.

Stiglitz, Joseph (1987): “Technological Change, Sunk Costs, and Competition”. En Brookings Papers on Economic Activity 3.

Sullivan, Lawrence A. (1977): Handbook of the Law of Antitrust. West Publishing.

Summers, L. (2001): "Competition Policy in the New Economy”. En Antitrust Law Journal, 69. 
United Kingdom Competition Commission (2003): “Merger References: Competition Commission Guidelines”. London.

U.S. Department of Justice and the Federal Trade Commission (1997): "Horizontal Merger Guidelines".

Von Weizsacker, C. C. (1980): “A Welfare Analysis of Barriers to Entry”. En Bell Journal of Economics, 11 (2).

Waterson, Michael (1984): Economic Theory of the Industry. Cambridge University Press.

Werden, Gregory J. (1998): “Demand Elasticities in Antitrust Analysis”. En Antitrust Law Journal, 66.

Werden, Gregory J. y Luke M. Froeb (1998): "The Entry-Inducing Effects of Horizontal Mergers: An Exploratory Analysis”. En Journal of Industrial Economics, 46.

Whinston, M. (2006): Lectures on Antitrust Economics. MIT Press.

Williamson, O. (1969), "Economies as an Antitrust Defense: The Welfare TradeOffs”. En American Economic Review, 18.

Willig, Robert D. (1991): "Merger Analysis, Industrial Organization Theory, and Merger Guidelines”. Brookings Papers on Economic Activity 281. 